\title{
Classification of Texture Images Based on the Histogram of Oriented Gradients Using Support Vector Machines
}

\author{
Hasan Demir \\ Department of Electronics and Communication Engineering, Namık Kemal University Çorlu School of Engineering, Tekirdağ, Turkey
}

Cite this article as: $\mathrm{H}$. Demir. "Classification of Texture Images Based on the Histogram of Oriented Gradients Using Support Vector Machines". Electrica, vol. 18, no. 1, pp. 90-94, 2018.

\begin{abstract}
Herein, using support vector machines, texture images were classified based on the histogram of oriented gradients, from which feature vectors were obtained. In addition, the success rate was examined for the feature vectors with different dimensions and the minimum length of a feature vector for performing classification was determined to be 288 elements.
\end{abstract}

Keywords: Texture classification, Support vector machines, Histogram of oriented gradients

Address for Correspondence:

Hasan Demir

\section{E-mail:}

hdemir@nku.edu.tr

Received: 20.12 .2017

Accepted: 04.01 .2018

(c) Copyright 2018 by Electrica

Available online at

http://dergipark.gov.tr/iujeee

DOI: $10.5152 /$ iujeee.2018.1814

\section{Introduction}

Texture analysis is the mostly used method in image processing. It is possible to get knowledge about segmentation and classification of spatial parameters in images by texture analysis. Texture analysis is frequently utilized in medical image processing, remote sensing, and control systems. Features of texture can be extracted with variety of methods such as statistics, geometry, model-based, and signal processing etc. [1-8]. Those features are classified by machine learning techniques such that support vector machines, artificial neural networks. Histogram of oriented gradients method has been primarily used for pedestarian detection [9]. In addition, this method has been used to solve problems about human detection, crowd detection, 3D segmentation, sign language recognition [10-13]. Support vector machines is proposed by Cortes and Vapnik for the purpose of solving two dimensional classification problem [14]. It can also be used to solve for multiclass classifying problem [15]. In addition, svm can be implemented for linear or nonlinear classification problems. In this study, hog and feature vector extraction will be discussed at first for texture images, and classification of feature vectors via support vector machine will be mentioned.

\section{Histogram of Oriented Gradients}

At first, image has been partitioned into piece of blocks and cells, and features of the image are obtained by calculating the gradient for every cell [9]. Therefore, images are represented with respect to local histograms. Vertical and horizontal gradient of the images are calculated with the help of Sobel filters. Lets assume $I(x, y)$ is the image, $f_{x}$ is horizontal gradient whose Sobel filter coefficients are [1.0,-1], and $f_{y}$ represents the vertical gradient whose Sobel filter coefficients are $[1,0,-1]^{\top} . I(x, y)$ represents the intensity of the image at $(x, y)$ point. Then gradient will be calculated as:

$f_{x}(x, y)=I(x+1, y)-I(x-1, y)$

$f_{y}(x, y)=I(x, y+1)-I(x, y-1)$ 
In addition,

$$
\begin{aligned}
& f(x, y)=\sqrt{f_{x}^{2}+f_{y}^{2}} \\
& \Theta=\arctan \frac{f_{y}(x, y)}{f_{x}(x, y)}
\end{aligned}
$$

where $f(x, y)$ represents the magnitude and $\Theta$ stands for the phase of the gradient.

\section{Support Vector Machines}

Support vector machines classify the group of data by using optimal hyperplane [14]. It is illustrated for 2-D data in Figure 1. Problem is to calculate $w$ and $b$ parameters with a constraint

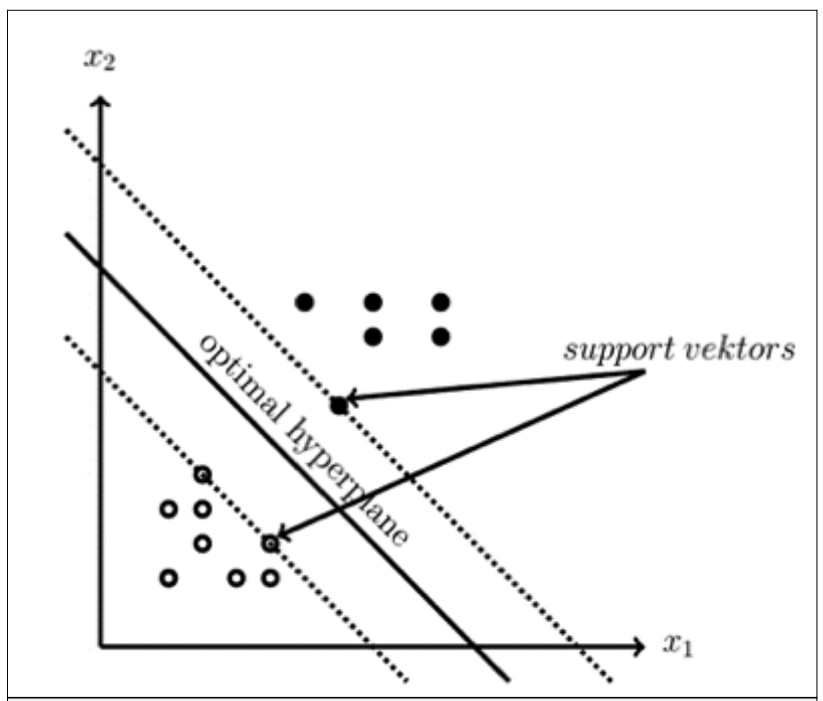

Figure 1. Support vectors and optimal hyperplane

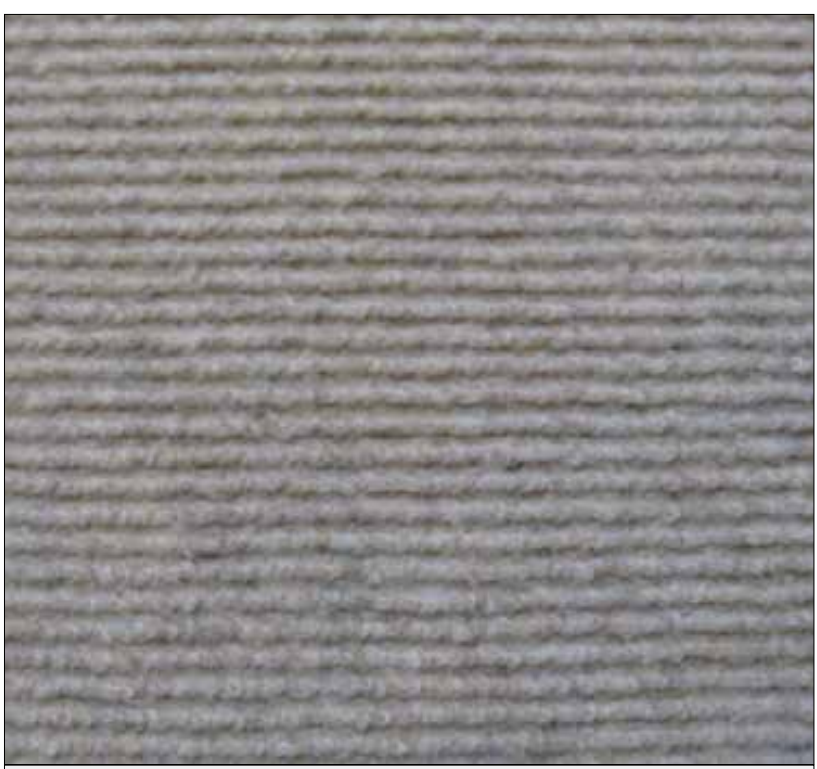

Figure 2. Texture image of fabric on optimum hyperplane such that $w x+b=0$, and $X_{i}$ can either be +1 or -1 .

\section{Texture Classification via HOG}

Texture data have been gathered from Salzburg Texture Image Database [16]. Size of the texture images are $512 \times 512$. Just three of the texture data: fabric, metal and tree textures are shown in Figure 2-4.

Implementation has been made by using Python programming language, and opencv machine learning library has been used. Texture images are converted into gray level images, and following methods are applied to those images.

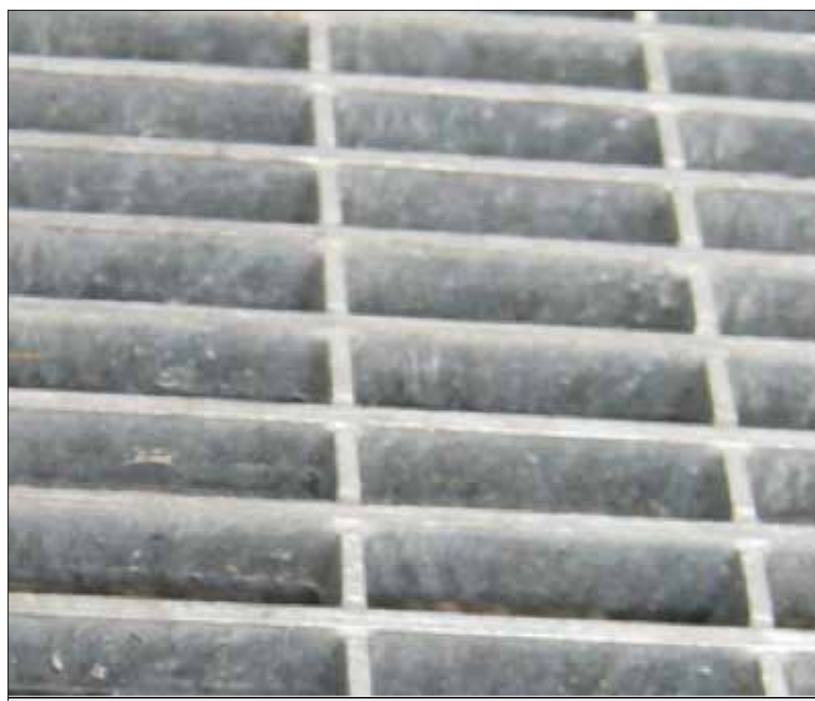

Figure 3. Texture image of metal

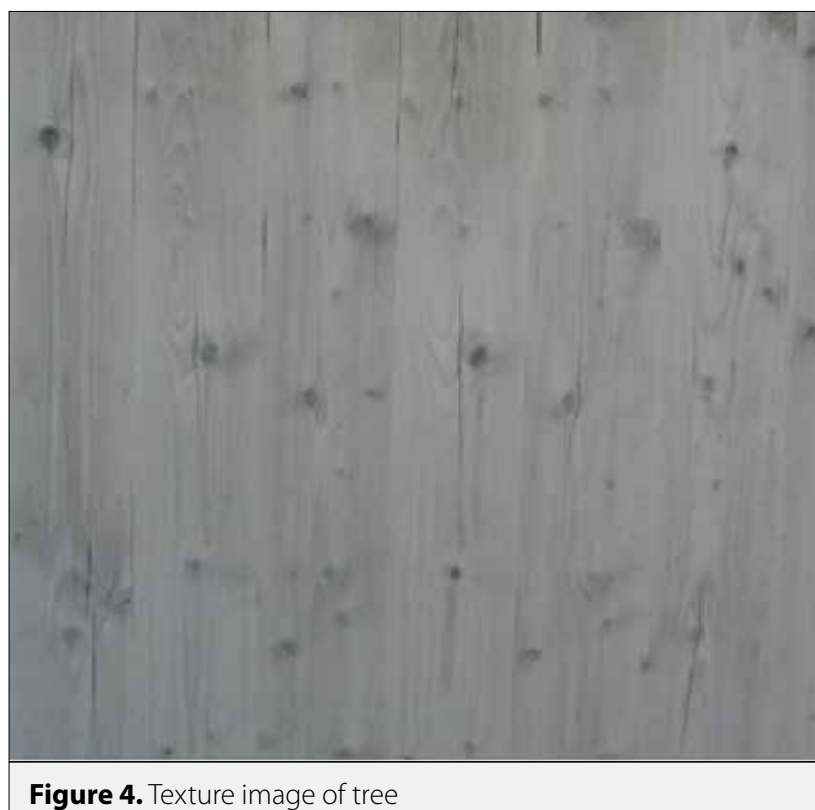




\section{Method 1}

Calculate the vertical and horizontal gradient for the images of size $512 \times 512$. Calculate amplitude and angle of the gradients. Histogram of angles are calculated by dividing [0.180) degree into 9 equal pieces. For every subimage, amplitude values are accumulated for each angle in the same piece. Therefore, $9 \mathrm{di}-$ mensional feature vector is obtained.

\section{Method 2}

Calculate the vertical and horizontal gradient for the images of size $512 \times 512$. Calculate amplitude and angle of the gradients. Histogram of angles are calculated by dividing $[-180,180)$ degree into 18 equal pieces. For every subimage, amplitude values are accumulated for each angle in the same piece. Therefore, 18 dimensional feature vector is obtained.

\section{Method 3}

Texture image is divided into four piece with a size of $256 \times 256$. Calculate vertical and horizontal gradient for these subimages. Calculate amplitude and angle of the gradients. Histogram of angles are calculated by dividing [0.180) degree into 9 equal pieces. For every subimage, amplitude values are accumulated for each angle in the same piece. Therefore, 9 dimensional feature vector is obtained. Concatenate all feature vectors together, and obtain $4 \times 9=36$ dimensional feature vector.

\section{Method 4}

Texture image is divided into four piece with a size of $256 \times 256$. Calculate vertical and horizontal gradient for these subimages. Calculate amplitude and angle of the gradients. Histogram of angles are calculated by dividing [-180, 180) degree into 18 equal pieces. For every subimage, amplitude values are accumulated for each angle in the same piece. Therefore, 18 dimensional feature vector is obtained. Concatenate all feature vectors together, and obtain $4 \times 18=72$ dimensional feature vector.

\section{Method 5}

Texture image is divided into 16 piece with a size of $128 \times 128$. Calculate vertical and horizontal gradient for these subimages. Calculate amplitude and angle of the gradients. Histogram of angles are calculated by dividing (0.180) degree into 9 equal pieces. For every subimage, amplitude values are accumulated for each angle in the same piece. Therefore, $9 \mathrm{di}-$ mensional feature vector is obtained. Concatenate all feature vectors together, and obtain 16×9=144 dimensional feature vector.

\section{Method 6}

Texture image is divided into four piece with a size of $128 \times 128$. Calculate vertical and horizontal gradient for these subimages. Calculate amplitude and angle of the gradients. Histogram of angles are calculated by dividing $[-180,180)$ degree into 18 equal pieces. For every subimage, amplitude values are ac-

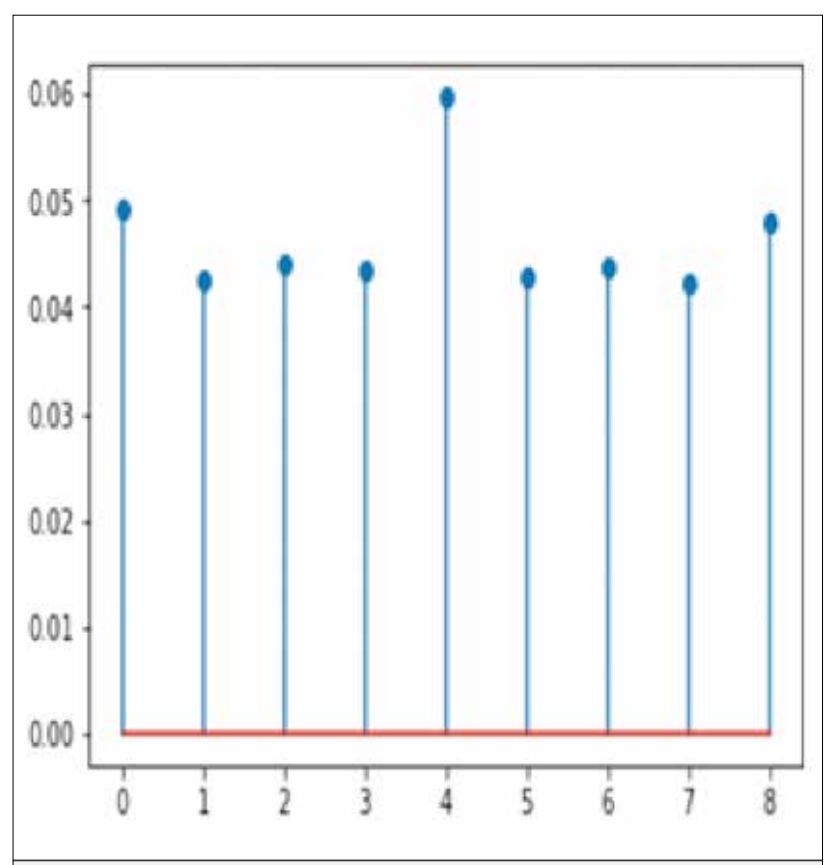

Figure 5. Feature vector calculated with Method 1.

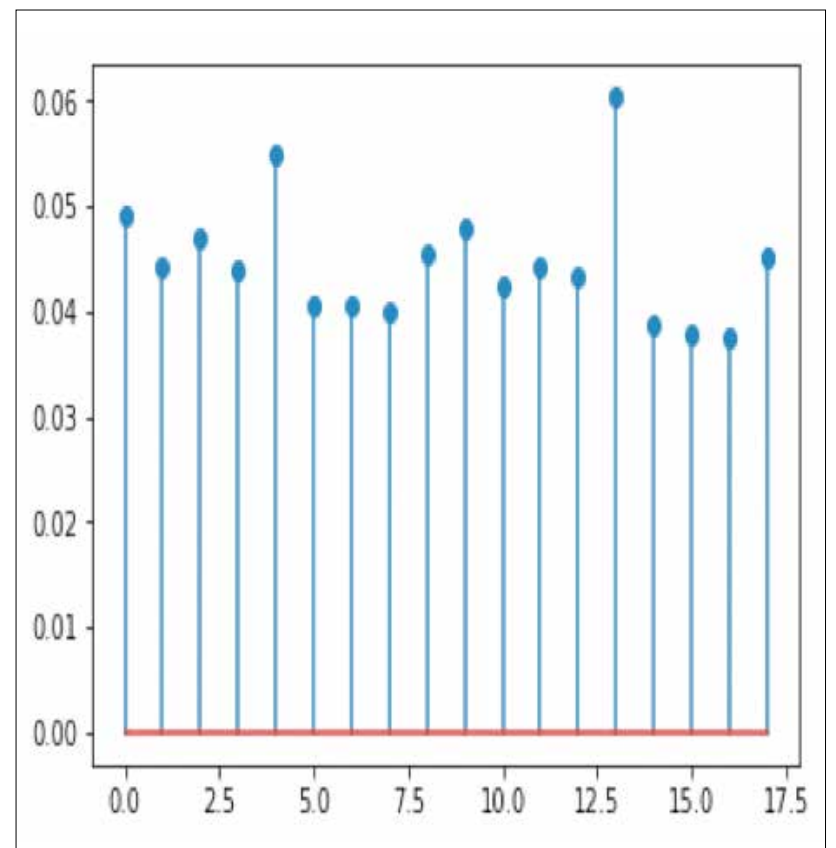

Figure 6. Feature vector calculated with Method 1.

cumulated for each angle in the same piece. Therefore, $18 \mathrm{di}-$ mensional feature vector is obtained. Concatenate all feature vectors together, and obtain 16x18=288 dimensional feature vector.

Normalized feature vectors are calculated with all the methods before mentioned for the texture image in Figure 2. In addition, all feature vectors are shown in Figure 5-10. 


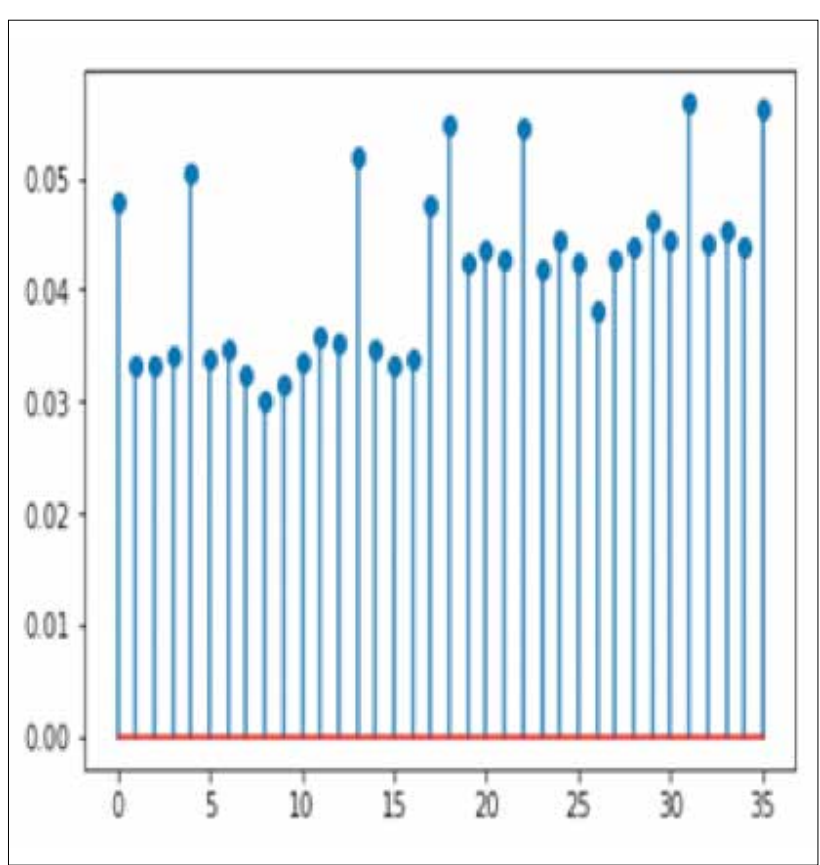

Figure 7. Feature vector calculated with Method 3

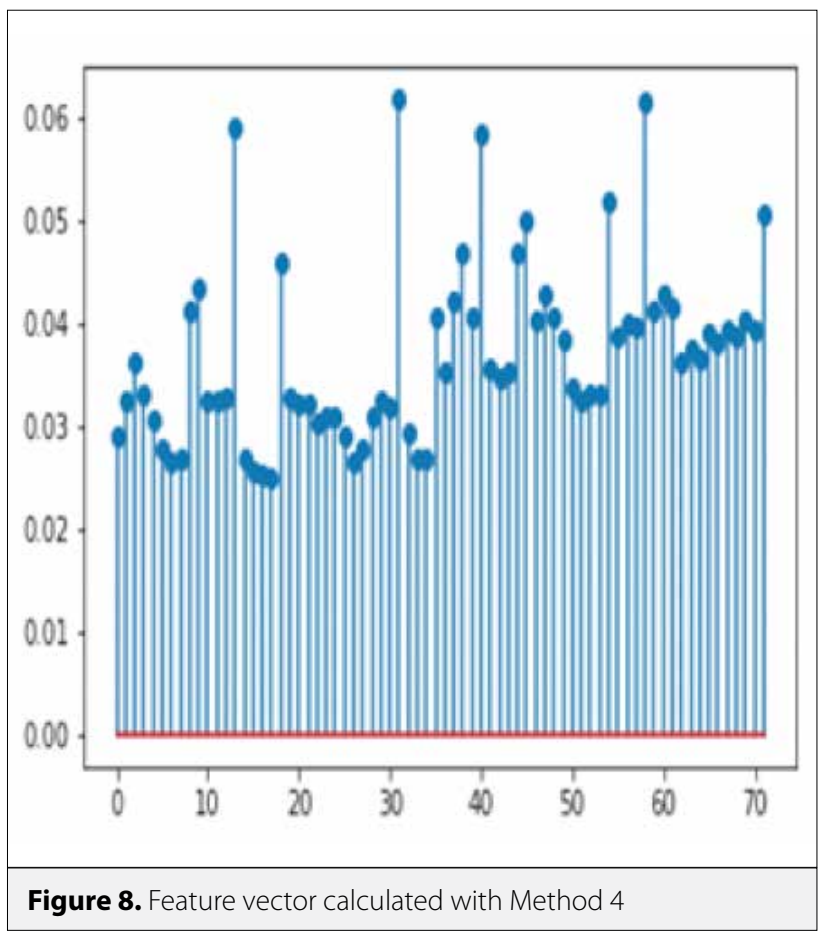

\section{Results and Discussion}

Opencv library has been used for the classification. Histogram intersection kernel is also utilized for the support vector machine. 230 of texture images which belong to 6 different class have been used as a training data, and 16 texture images have been utilized as a testing data. Success rate of classification are given in Table 1.

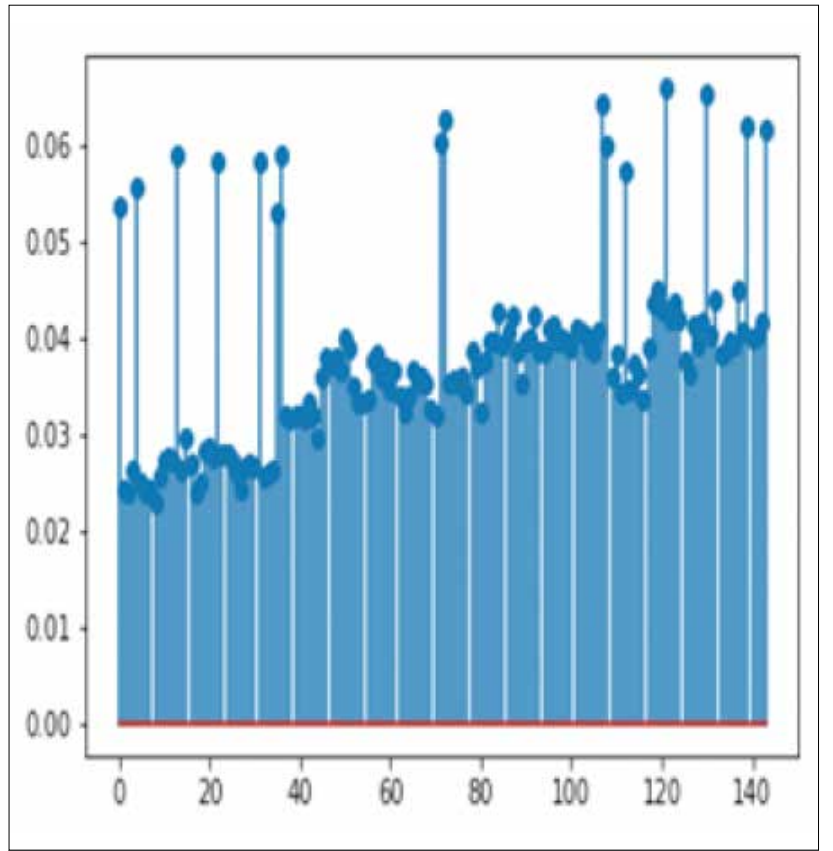

Figure 9. Feature vector calculated with Method 5

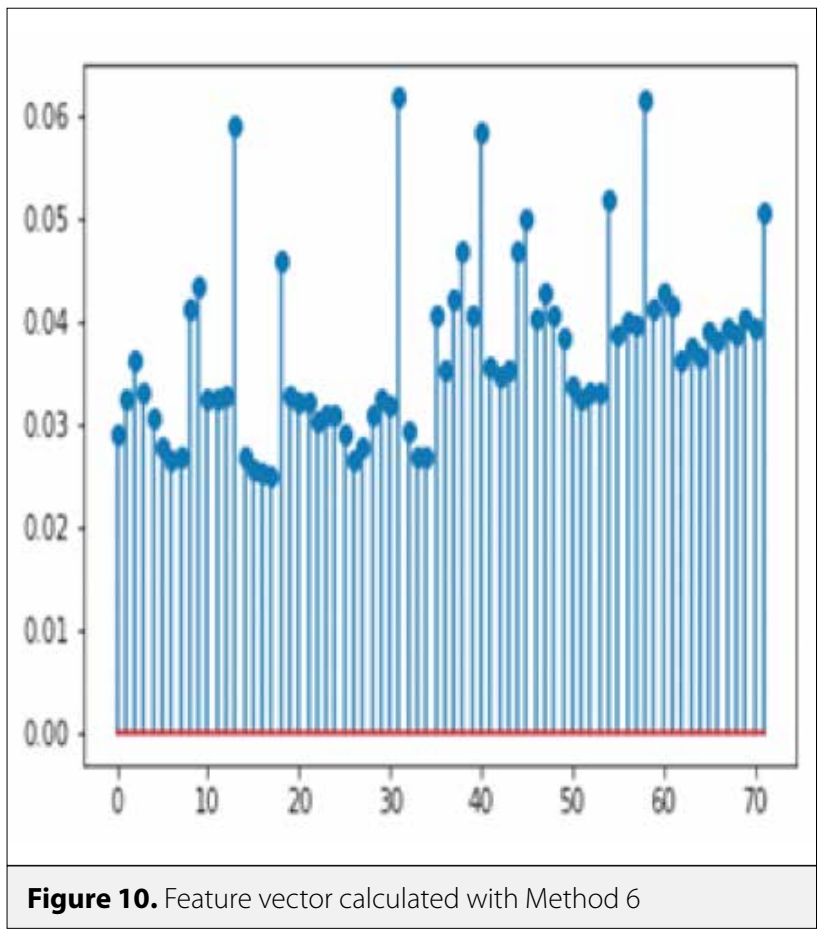

In the literature, size of feature vector using histogram of oriented gradients have been reported 3780 elements [18]. In the method 6, size of feature vector has obtained 288 elements.

\section{Conclusions}

Methods which are related with generating 18 piece of histograms with an angles between $[-180,180)$ have better perfor- 
Table 1. Success rates of classification

\begin{tabular}{lc}
\hline Method & Success rate \\
\hline 1 & $43.75 \%$ \\
\hline 2 & $25 \%$ \\
\hline 3 & $50 \%$ \\
\hline 4 & $62.25 \%$ \\
\hline 6 & $31.25 \%$ \\
\hline
\end{tabular}

mance results than other methods, i.e Method 4 and Method 6 . For those methods consisting of 18 piece of histograms have better success rates when the numbers of feature vectors are increased. Method 6 is the best method when success rates are taken into account.

\section{References}

1. M. Tuceryan, A. K. Jain, "Texture analysis - Handbook of Pattern Recognition \& Computer Vision," World Scientific Pub Co Inc., Singapore City, 1993.

2. D. Marr, “Vision”, Freeman, Chap 2, pp. 54-78, 1982.

3. H. Voorhees, T. Poggio, “Detecting textons and texture boundaries in natural images", Proceedings of the First International Conference on Computer Vision. pp 250-258, 1987.

4. M. Tuceryan, A. K. Jain, "Testure segmentation using Voronoi polygons," IEEE Transactions on Pattern Analysis and Machine intelligence vol. 12, pp. 211-216, 1990. [CrossRef]

5. F. Tomita, S. Tsuji, "Computer Analysis of Visual Textures," Springer, New York, 1990. [CrossRef]

6. L. S. Davis, S. A. Johns, J. K. Aggarwal, "Texture Analysis Using Generalized Co-Occurrence Matrices," IEEE Transactions on Pattern Analysis and Machine Intelligence, vol. PAMI-1, Issue: 3, July 1979. [CrossRef]
7. N. Ahuja, B. J. Schachter, "Pattern Models", John Wiley, 1983.

8. M. Clark, A. C. Bovik, W. S. Geisler, "Texture Segmentation Using Gabor Modulation/Demodulation," Pattern Recognition Letters, vol. 6, pp. 261-267, September 1987. [CrossRef]

9. N. Dalal, B. Triggs. "Histograms of Oriented Gradients for Human Detection", IEEE Computer Society Conference on Computer Vision and Pattern Recognition, vol.1, pp. 886-893, June 2005. [CrossRef]

10. T. Watanabe, S. Ito, K. Yokoi, "Image Feature Descriptor using Co-occurrence Histograms of Oriented Gradients for Human Detection," The Journal of the Institute of Image Information and Television Engineers, vol. 71, pp. J28, 2017. [CrossRef]

11. H. Fu, H. Ma, "Real-time crowd detection based on gradient magnitude entropy model," Proceedings of the ACM International Conference on Multimedia, November 03-07, 2014, Orlando, Florida, USA. [CrossRef]

12. C. Bhole, N. Morsillo, C. Pal, " $3 \mathrm{D}$ segmentation in CT imagery with conditional random fields and histograms of oriented gradients", Proceedings of the Second international conference on Machine learning in medical imaging, p. 326-334, September 18, 2011, Toronto, Canada. [CrossRef]

13. P. Jangyodsuk, C. Conly, V. Athitsos, "Sign language recognition using dynamic time warping and hand shape distance based on histogram of oriented gradient features", Proceedings of the 7th International Conference on PErvasive Technologies Related to Assistive Environments, May 27-30, 2014, Rhodes, Greece. [CrossRef]

14. C. Cortes, V. Vapnik, "Support-Vector Networks", Kluwer Academic Publishers Machine Learning, vol. 20, pp. 273-297, 1995. [CrossRef]

15. A. Wang, W. Yuan, J. Liu, Z. Yu, H. Li, "A novel pattern recognition algorithm: Combining ART network with SVM to reconstruct a multi-class classifier," Computers and Mathematics with Applications, vol. 57, pp. 1908-1914, 2009. [CrossRef]

16. R. Kwitt, P. Meerwald, "Salzburg texture image database," Available online: http://www.wavelab.at/sources/STex/

17. A. Barla, F. Odone, A.Verri "Histogram intersection kernel for image classification" Image Processing, 2003. ICIP 2003. Proceedings. 2003 International Conference on, vol. 3.

18. C.Tomasi,"Histograms of Oriented Gradients", http://www.cs.duke. edu/courses/fall15/compsci527/notes/hog.pdf, 2017.- Accessed: 20.11.2017 tronics and Communication Engineering Department in 1994, Master's Degree in from Institute of Science, İstanbul University in 1998, and his Ph.D. degree from İstanbul University, Institute of Science in 2008. Between 1994 and 1998, He worked as an assistant at T.Ü. Çorlu Engineering Faculty, and between 1998-2008 he worked as a research assistant in the Department of Electrical and Electronics Engineering at the I.U. Faculty of Engineering. Currently, he is an Assistant Professor in the Department of Electronics and Communication Engineering at Çorlu Engineering Faculty, N.K.U. He works on artificial neural networks, image processing, wavelet analysis. 